\title{
IDEAL MULTIPLICATION IN A LINEAR ALGEBRA*
}

\author{
BY GRACE SHOVER AND C. C. MACDUFFEE
}

1. Introduction. A recent approach $\dagger$ to the theory of ideals through matrices with rational integral elements proved to be successful for domains of integrity of linear associative algebras over the rational field as well as for domains of algebraic fields. The great weakness of this method was the lack of an adequate treatment of ideal multiplication.

In the present paper it is shown that ideal multiplication can be treated in a satisfactory manner by means of the matric theory. The way now seems to open for considerable development of the theory of ideals in rational algebras.

It is particularly to be noted that the classical theory of ideals in an algebraic field is a special case of the present theory. By way of illustration it is shown that the method of this paper can be applied to find by a straightforward, rational process the canonical form of the product of two ideals.

2. Definitions and References. Let $\mathfrak{A}$ be a rational semi-simple algebra of order $n$, and $\mathfrak{S}$ a set of integral elements of order $n$ in $\mathfrak{A} . \ddagger$ We suppose that the basal numbers $e_{1}, e_{2}, \cdots, e_{n}$ of $\mathfrak{A}$ form a basis for $\Im, e_{1}$ being the principal unit.

If the constants of multiplication are $c_{i j k}$, that is, if

then the matrices

$$
e_{i} e_{j}=\sum_{k} c_{i j k} e_{k} \quad(i, j=1,2, \cdots, n),
$$

$$
R_{i}=\left(c_{i s r}\right), S_{i}=\left(c_{r i s}\right)
$$

are called the first and second matrices $\S$ of $e_{i}$. If $\xi=\sum x_{i} e_{i}$ is the general number, then

$$
R(\xi)=\sum x_{i} R_{i}, \quad S(\xi)=\sum x_{i} S_{i}
$$

form sets of matrices each of which gives a matric representation of the algebra.

\footnotetext{
* Presented to the Society, December 30, 1930.

$\dagger$ C. C. MacDuffee, Transactions of this Society, vol. 31 (1929), pp. 71-90.

$\ddagger$ Dickson, Algebren und ihre Zahlentheorie, 1927, p. 155.

$\S$ MacDuffee, this Bulletin, vol. 35 (1929), p. 344.
} 
A left (right) ideal $\Omega$ is a set of numbers of $\subseteq$ which is closed under addition and subtraction, and under multiplication on the left (right) by the numbers of $\Im$. If not every number of $\Re$ is of norm* $0, \Omega$ is non-singular. We shall speak only of left ideals, as the theory of right ideals is obviously parallel.

Let $\Omega_{\alpha}$ be an ideal with basis $\left(\alpha_{1}, \alpha_{2}, \cdots, \alpha_{n}\right)$. Then

$$
\alpha_{i}=\sum_{j=1}^{n} g_{i j} e_{j}
$$

where the $g_{i j}$ are rational integers. We shall call $G_{\alpha}=\left(g_{r s}\right)$ an ideal matrix corresponding* to the ideal $\mathfrak{\Omega}_{\alpha}$. If $\left|g_{r s}\right| \neq 0$, then $\Omega_{\alpha}$ is non-singular. If $G_{\alpha}$ corresponds to the non-singular ideal $\Omega_{\alpha}$, then the totality of ideal matrices corresponding to $\Re_{\alpha}$ is given by $A G_{\alpha}$, where $A$ is a matrix with rational integral elements (in brief, an integral matrix) of determinant \pm 1 .

If $\Omega_{\alpha}$ and $\Omega_{\beta}$ are two ideals, we define the ideal product $\Omega_{\alpha} \Omega_{\beta}$ to be the set of numbers

$$
\delta=\sum_{i, j=1}^{n} d_{i j} \alpha_{i} \beta_{j}
$$

where the $d_{i j}$ range over all rational integers. That this set is an ideal follows immediately, for if $\xi$ is a number of $\subseteq$,

$$
\xi \delta=\sum_{i, j} d_{i j} \xi \alpha_{i} \beta_{j}
$$

But

$$
\xi \alpha_{i}=\sum_{h} e_{i h} \alpha_{h}
$$

where the $e_{i n}$ are rational integers, since $\alpha_{1}, \cdots, \alpha_{n}$ form a basis for the ideal $\mathfrak{\Omega}_{\alpha}$. Thus

$$
\xi \delta=\sum_{i, j, h} d_{i j} e_{i h} \alpha_{h} \beta_{j}
$$

which is again a member of the set. We shall denote this product ideal by $\Omega_{\pi}$. Note that ideal multiplication is not always commutative.

* MacDuffee, Transactions of this Society, loc. cit., p. 74. 
Du Pasquier* has proved that if $M$ and $N$ are two integral square matrices of order $n$, not both singular, they possess a greatest common right divisor

$$
D=P M+Q N
$$

and that every g. c. r. d. of $M$ and $N$ is given by $A D$ where $A$ is an integral matrix of determinant \pm 1 . Thus the g. c. r. d. has the same latitude of definition as an ideal matrix. The theorem extends readily to $n$ matrices, and du Pasquier shows how $D$ may be readily computed.

3. Theorem. If the ideals $K_{\alpha}$ and $K_{\beta}$ are represented by the matrices $G_{\alpha}$ and $G_{\beta}$ respectively, then every matrix representing the product $K_{\pi}=K_{\alpha} K_{\beta}$ is a greatest common right divisor of the $n$ matrices

$$
G_{\alpha} S\left(\beta_{i}\right), \quad(i=1,2, \cdots, n),
$$

and also of the matrices

$$
G_{\beta} \bar{R}\left(\alpha_{i}\right), \quad(i=1,2, \cdots, n),
$$

where $\bar{R}$ is the transpose of $R$.

Suppose that

$\alpha_{i}=\sum a_{i j} e_{j}, \beta_{i}=\sum b_{i j} e_{j}, \pi_{i}=\sum p_{i j} e_{j},(i, j=1,2, \cdots, n)$.

Since every number of $K_{\alpha} K_{\beta}$ is in $K_{\pi}$, there exist in particular rational integers $h_{i j k}$ such that

$$
\alpha_{i} \beta_{i}=\sum_{k=1}^{n} h_{i j k} \pi_{k}, \quad(i, j=1,2, \cdots, n) .
$$

That is,

$$
\sum_{k, l, m} a_{i k} b_{j l} c_{k l m} e_{m}=\sum_{k, m} h_{i j k} p_{k m} e_{m} .
$$

On account of the linear independence of the basal numbers,

$$
\sum_{k, l} a_{i k} b_{j l} c_{k l m}=\sum_{k} h_{i j k} p_{k m}, \quad(i, j, m=1,2, \cdots, n),
$$

* Vierteljahrsschrift der Naturforschenden Gesellschaft in Zürich, vol. 51 (1906), p. 89. 
which can be written in either of the two forms

$$
\begin{aligned}
\sum_{k} a_{r k}\left(\sum_{l} b_{j l} c_{k l s}\right) & =\sum_{k} h_{r j k} p_{k s}, \\
\sum_{l} b_{r l}\left(\sum_{k} a_{i k} c_{k l s}\right) & =\sum_{k} h_{i r k} p_{k s} .
\end{aligned}
$$

In matric notation the first of these equations is

$$
G_{\alpha} S\left(\beta_{j}\right)=H_{1 j} G_{\pi}, \quad H_{1 j}=\left(h_{r j s}\right),
$$

and the second is

$$
G_{\beta} \bar{R}\left(\alpha_{i}\right)=H_{2 i} G_{\pi}, \quad H_{2 i}=\left(h_{i r 8}\right) .
$$

Thus $G_{\pi}$ is a common right divisor of the matrices $G_{\alpha} S\left(\beta_{j}\right)$, and also of the matrices $G_{\beta} \bar{R}\left(\alpha_{i}\right)$.

Since every number of $K_{\pi}$ is in $K_{\alpha} K_{\beta}$, there exist rational integers $k_{i j h}$ such that

That is,

$$
\pi_{i}=\sum_{j, h} k_{i j h} \alpha_{j} \beta_{h}, \quad(i=1,2, \cdots, n) .
$$

$$
\sum_{t} p_{i t} e_{t}=\sum_{j, h, l, m, t} k_{i j h} a_{\jmath l} b_{h m} c_{l m t} e_{t}
$$

whence

$$
p_{i t}=\sum_{j, h, l, m} k_{i j h} a_{j l} b_{h m} c_{l m l},(i, t=1,2, \cdots, n) .
$$

This may be written in either of the forms

$$
\begin{aligned}
& p_{r s}=\sum_{j, h, l} k_{r j h} a_{j l} \sum_{m} b_{h m} c_{l m s}, \\
& p_{r s}=\sum_{j, h, m} k_{r j h} b_{h m} \sum_{l} a_{j l} c_{l m s} .
\end{aligned}
$$

In matric form these equations are, respectively,

$$
\begin{array}{rlrl}
G_{\pi} & =\sum_{h} K_{1 h} G_{\alpha} S\left(\beta_{h}\right), & K_{1 h} & =\left(k_{r s h}\right), \\
G_{\pi} & =\sum_{j} K_{2 j} G_{\beta} \bar{R}\left(\alpha_{j}\right), & K_{2 j}=\left(k_{r j s}\right) .
\end{array}
$$

Thus $G_{\pi}$ is a greatest common right divisor of the matrices $G_{\alpha} S\left(\beta_{h}\right)$ and also of the matrices $G_{\beta} \bar{R}\left(\alpha_{j}\right)$. 
If the ideals $K_{\alpha}$ and $K_{\beta}$ are non-singular, we may assume that not every $\alpha_{i}$ and not every $\beta_{i}$ is of norm 0 . In particular $K_{\alpha}$ and $K_{\beta}$ may have canonical bases, in which case $\alpha_{1}$ and $\beta_{1}$ are the smallest positive integers in the respective ideals. Thus not every $S\left(\beta_{i}\right)$ and not every $\bar{R}\left(\alpha_{i}\right)$ is singular. Since $G_{\alpha}$ and $G_{\beta}$ are non-singular matrices, the matrix $G_{\pi}$ is unique up to a left factor $A$ which is an integral matrix of determinant \pm 1 .

4. Canonical Form of an Ideal Product. We shall indicate just one application of the preceding theorem. The problem of obtaining the canonical form of the product ideal from the canonical forms of the factors has been discussed for quadratic fields, ${ }^{*}$ but not for the general case.

By the method of $\S 3$ the matrix $G_{\pi}$ is obtained directly by du Pasquier's method when $G_{\alpha}$ and $G_{\beta}$ are non-singular, that is, in the only case considered for algebraic fields. Then by a theorem due to Hermite $\dagger$ we can, by multiplying $G_{\pi}$ on the left by an integral matrix $A$ of determinant \pm 1 , obtain the canonical matrix

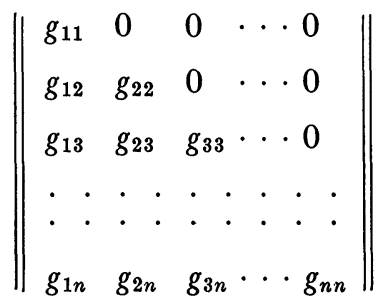

in which every $g_{i j} \geqq 0, g_{i i}>0$, and each element in the $i$ th column below the main diagonal is reduced modulo $g_{i i}$. Moreover this canonical form is unique.

All the steps in this reduction are mechanical and involve working only with rational integers.

Connecticut College, and Ohio State University 87.

* W. B. Carver, American Mathematical Monthly, vol. 18 (1911), pp. 81-

† Journal für Mathematik, vol. 41 (1851), p. 193. 